\title{
Lower hybrid current drive in the presence of electric field
}

\author{
Alexander Saveliev ${ }^{1, *}$, and Vladimir Zakharov ${ }^{1}$ \\ ${ }^{1}$ Ioffe Institute, Plasma Physics Laboratory, 194021 St. Petersburg, Russia
}

\begin{abstract}
A new one-dimensional approach to the lower hybrid current drive (LHCD) modelling in the presence of an inductive electric field is suggested in this paper. The approach is based on using timedependent solutions of a well-known Fokker-Planck equation for the distribution function of fast electrons calculated concurrently with solving plasma transport equation in the Automated System for TRansport Analysis (ASTRA) [1]. A good agreement between experimental and modelling results is demonstrated for an FT-2 [2] plasma shot. Also new formulae for the steady-state solution of this kinetic equation are found.
\end{abstract}

\section{Analytical analysis}

The well-known steady-state solution for the onedimensional distribution function of fast electrons in the absence of an electric field [3] is still used for the lower hybrid current drive modelling because of its simplicity combined with a rather good calculated driven current accuracy [4]. Actually, the electric field is only absent in a steady-state situation in which the plasma current is entirely driven by the waves. In most LHCD experiments the inductive electric field is present and can play a noticeable role in the LH current generation. In this paper we describe a steady-state solution of the 1D kinetic equation for the fast $\left(V_{\|} / V_{T_{e}} \gg 1\right)$ electrons and discuss application of the solution to the lower hybrid (LH) current drive simulations. This equation has the following form in standard notations [5].

$\frac{\partial f}{\partial \tau}=E \frac{\partial f}{\partial v}+\frac{\partial}{\partial v}\left\{D(v) \frac{\partial f}{\partial v}\right\}+\beta\left(Z_{e f f}\right) \frac{\partial}{\partial v}\left\{\frac{1}{v^{3}} \frac{\partial f}{\partial v}+\frac{f}{v^{2}}\right\}$

Here $D(v)$ is the quasilinear diffusion coefficient that represents the effect of the waves, $v=V_{\|} / V_{T_{e}}, V_{\mathrm{Te}}=$ $\sqrt{T_{e} / m_{e}}, E=E_{\|} / E_{c}, \quad E_{c}=4 \pi e^{3} n_{e} L / T_{e}, L$ is the Coulomb logarithm and time is normalized to the Spitzer collision time $\tau=t / \tau_{0}, \tau_{0}=m_{e}^{2} V_{T_{e}}^{3} / 4 \pi e^{3} n_{e} L$. To reconcile the $1 \mathrm{D}$ solution with the prediction of the $2 \mathrm{D}$ theory we introduce a parameter $\beta=\left(5+Z_{\text {eff }}\right) / 5$, which actually means renormalization of the Spitzer collision frequency. The equation (1) includes the runaway problem leading to a divergence of the electron distribution function (EDF) wing $f_{a c c}(v)$ where the electric field $E$ accelerates electrons. Nevertheless, for small normalized parallel electric field $|E| \ll 1$ the runaway problem can be solved as stationary [6] because a very large number of electrons in the Maxwellian body of the distribution function play the role of a large reservoir feeding a small stationary electron flux in the direction of higher energies. Following the methods given in [6] and [7], we found for the $f_{a c c}(v)$ a steadystate solution $\left(\frac{\partial f}{\partial \tau}=0,|E| \ll 1\right)$ of the equation (1) in the form of integrals of elementary functions.

$$
\begin{aligned}
f_{a c c}(v) & =\frac{1}{\sqrt{2 \pi} C} \int_{v}^{\infty} \frac{x^{3}}{1+D x^{3}} \exp \left(\int_{v}^{x} \frac{t-|E| t^{3}}{1+D t^{3}} d t\right) d x \\
\boldsymbol{C} & =\int_{0}^{\infty} \frac{x^{3}}{1+D x^{3}} \exp \left(\int_{0}^{x} \frac{t-|E| t^{3}}{1+D t^{3}} d t\right) d x
\end{aligned}
$$

For the wing of the distribution function $f_{d e c}(v)$ where the electric field decelerates electrons we obtain the following solution of the equation (1) for $\frac{\partial f}{\partial \tau}=0$ and an arbitrary absolute value of the electric field $|E|$ :

$$
f_{\text {dec }}(v)=\frac{1}{\sqrt{2 \pi}} \exp \left[-\int_{0}^{v}\left(\frac{t+|E| t^{3}}{1+D t^{3}}\right) d t\right]
$$

It should be noted that the solution (2) in contrast to the solution (3) has a nonzero flux of fast electrons propagating in the direction of higher energies. In the particular case $D(v)=0$, integrals in (2) can be calculated analytically. This simplifies solution (2) to a combination of the probability integral and elementary functions.

$$
f_{a c c}(v)=\frac{e^{-\frac{1}{4|E|}}}{\sqrt{2 \pi}}\left(\frac{e^{x^{2} \psi(x)+2 \sqrt{\frac{|E|}{\pi}}}}{2-\Psi\left(\frac{1}{2 \sqrt{|E|}}\right)+2 \sqrt{\frac{|E|}{\pi}} e^{-\frac{1}{4|E|}}}\right)
$$

Here the following notations are used:

$$
\begin{gathered}
x=\frac{1}{2}\left(v^{2} \sqrt{|E|}-1 / \sqrt{|E|}\right) \\
\Psi(x)=1-\Phi(x) ; \quad \Phi(x)=\frac{2}{\sqrt{\pi}} \int_{0}^{x} e^{-t^{2}} d t
\end{gathered}
$$

This formula (4) is a new one. Then, for the decelerating electrons and $D(v)=0$ we easily obtain from (3) a simple formula (5).

$$
f_{\text {dec }}(v)=\frac{1}{\sqrt{2 \pi}} \exp \left(-\frac{v^{2}}{2}-\frac{|E| v^{4}}{4}\right)
$$




\section{Numerical analysis}

\subsection{Zero electric field}

We start with an example of the LH driven current profiles calculated for the case of $\mathrm{E}=0$ by a stand-alone version of the Fast Ray-Tracing Code (FRTC) [8, 9] for the FT-2 plasma parameters of the shot \#041514_35 at the time slice $\mathrm{t}=0.032$ seconds and $32 \mathrm{kA}$ of plasma current. The simulation result is given in Fig. 1.
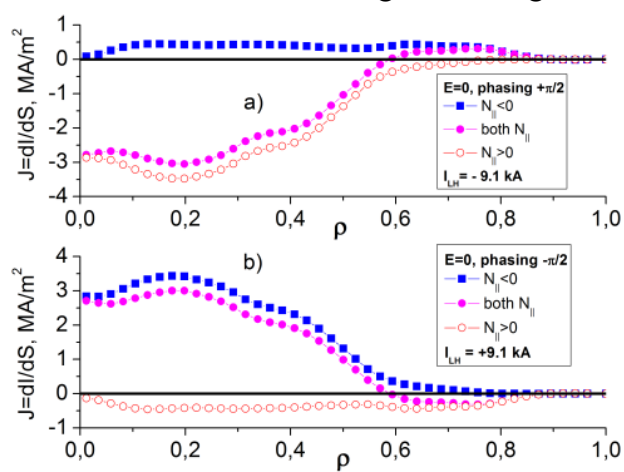

Fig. 1. Driven current profiles calculated by FRTC with $E=0$. a) antenna phasing is $+\pi / 2$; b) antenna phasing $-\pi / 2$.

Initial $N_{\|}$spectra for the FT-2 LH two-waveguide antenna noticeably differs from the standard LH spectra because its positive and negative lobes contain comparable amounts of the LH input power. Examples of the FT-2 spectra [10] calculated for opposite waveguide phasing is present in Fig. 2.

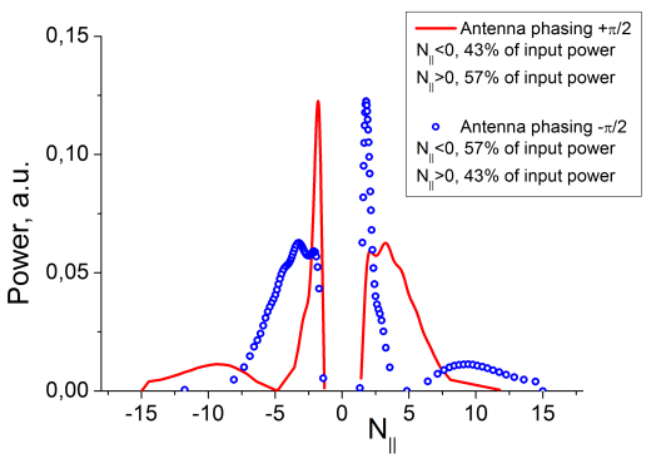

Fig. 2. Calculated initial FT-2 $N_{\|}$spectra for $+\pi / 2$ and $-\pi / 2$ phasing of the FT- 2 antenna.

Predicted driven currents in Fig. 1 for the case of the inductive electric field absence turns out oppositely directed for the $+\pi / 2$ and $-\pi / 2$ antenna phasing's. It must considerably influence the loop voltage, which is never the case in the FT-2 LHCD experiments, where changing phasing from $+\pi / 2$ to $-\pi / 2$ never changed the sign of the loop voltage. This weak dependence of the loop voltage on the antenna phasing can be explained by the influence of the inductive electric field on the LH current generation. Namely, the electric field enhances $f_{a c c}(v)$ and suppresses the $f_{\text {dec }}(v)$ wings of the distribution function, leading to an additional asymmetry of the EDF, which may strongly change driven current profile and value. The other part of the paper is devoted to the LHCD modelling with $|E|>0$.

\subsection{Nonzero electric field}

Numerical solution of the Fokker-Planch equation (1) with initial Maxwellian distribution function and typical profile of the quasilinear diffusion coefficient $D(v)$ given in Fig. 3 showed that the steady-state distribution function of fast electrons (2) is reached at time $t_{\text {asymp }}$, which is approximately equal to ten collision times $t_{s t\left(4 V_{T e}\right)}$ of fast electrons $\left(V_{\|}=4 V_{T_{e}}\right.$, where the quasilinear plateau starts) with the thermal electrons.

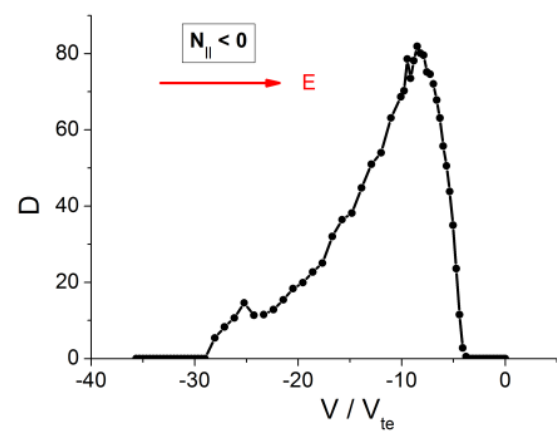

Fig. 3. Typical $D(v)$ profile obtained by the FRTC.

In Fig. 4 you can see evolution of the $f_{a c c}(v)$ calculated for $|E|=0.04$ and $D(v)$ given in Fig. 3 .

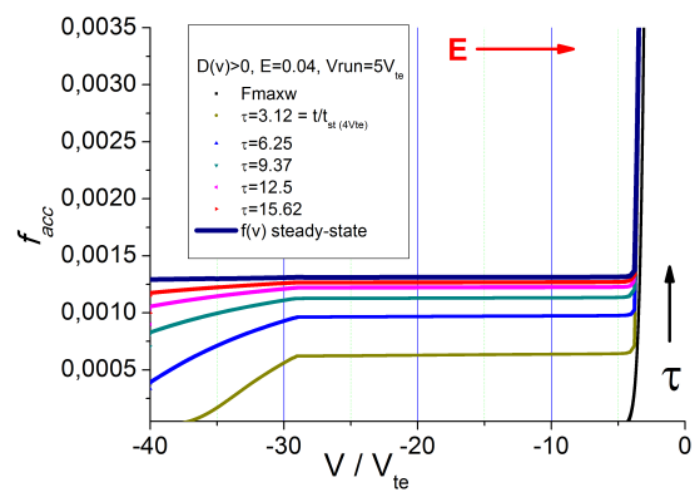

Fig. 4. $f_{a c c}(v)$ evolution with time $\tau=t / t_{s t\left(4 V_{T e}\right)}$

Note, that $t_{\text {asymp }}$ depends mainly on the quasilinear plateau's width and is almost independent of the electric field value. This statement is illustrated by the Fig. 5 .

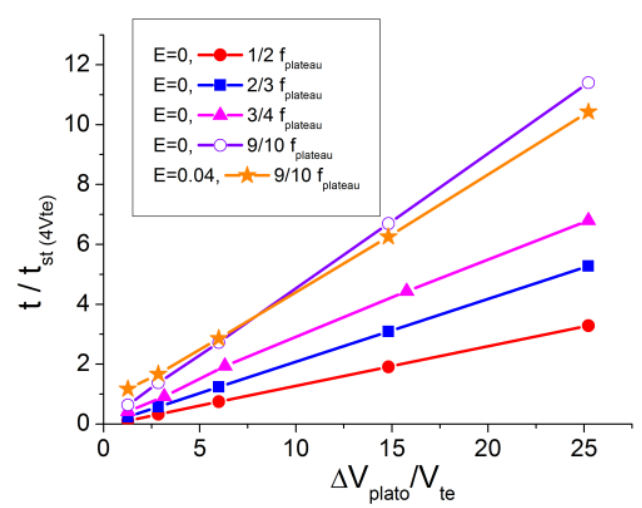

Fig. 5. Normalized times needed to reach different levels of the steady-state $\operatorname{EDF} f_{a c c}(v):\left(1 / 2 \mathrm{f}_{\text {plateau }}, 2 / 3 \mathrm{f}_{\text {plateau, }}\right.$, etc. $)$. 
A few dependences of $t_{\text {asymp }}$ on plasma temperature and density for $\beta\left(Z_{e f f}\right)=1$ are given in Fig. 6 . For $\beta \neq 1$ the $t_{\text {asymp }}$ should be divided by $\beta$.

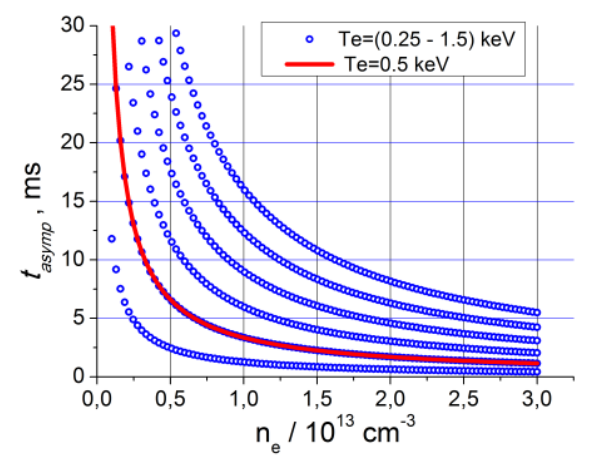

Fig. 6. $t_{\text {asymp }}$ as a function of plasma temperature and density. Each curve corresponds to a fixed temperature on the equidistant greed in the interval $0.25 \div 1.5 \mathrm{keV}$.

It is seen from the Fig. 6 that $t_{\text {asymp }}$ may vary across the minor tokamak radius from a few to a few dozen milliseconds, so the formulae (2-5) for the steady-state distribution function can be, in principle, used in the LHCD modelling of experiments with much longer LH impulse duration than the $t_{\text {asymp }}$. For small tokomaks Globus-M, Globus-M2 and FT-2 the LH impulse duration is comparable with the $t_{\text {asymp }}$ which makes the LHCD simulation task nonstationary for these tokamaks. Moreover, the application of the stationary solution (2-5) for the distribution function of fast electrons in modelling of the FT-2 LHCD experiments gives obviously wrong result in many cases, namely the overestimated driven current. An example of the steadystate formulae (2-5) application is given in Fig. 7, where you can see the LH driven current profiles calculated by a stand-alone FRTC version for the same plasma conditions as in Fig. 1 and the electric field radial profile taken from the ASTRA run.

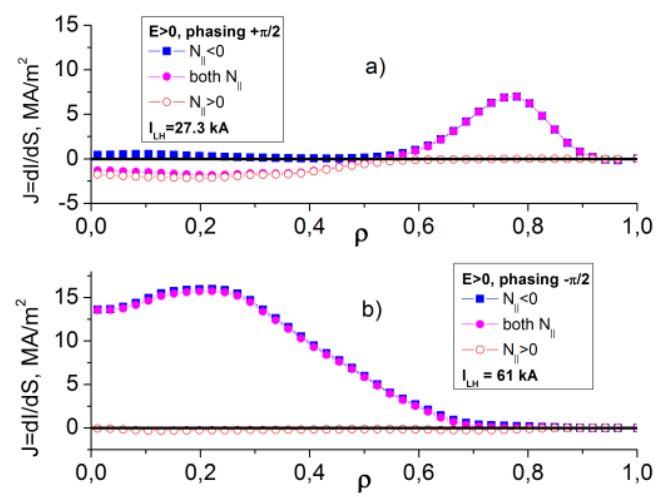

Fig. 7. Driven current profiles calculated by FRTC using steady-state formulae (2-5) for the EDF, $|E|>0$. a) antenna phasing is $+\pi / 2, \mathrm{~b}$ ) reverse antenna phasing $-\pi / 2$.

It is seen from the Fig. 7 that calculated LH driven current for $+\pi / 2$ antenna phasing is almost equal to the total plasma current, which should lead to almost zero loop voltage, but it is not the case in this experiment (see Fig. 8). Results of modelling for $-\pi / 2$ antenna phasing are even more far from the all FT-2 experimental experience. So, we have to make a conclusion that formulae (2-5) lead to driven current overestimation for the FT-2 experimental conditions. To explain this disagreement between experiment and modelling we supposed that distribution function for the fast electrons does not have enough time to reach the steady-state level described by the formulae (2-5), because duration of the LH power impulse is only 6 milliseconds in the FT-2 experiments which is almost equal to the corresponding value of the $t_{\text {asymp }}$. Therefore the EDF time evolution must be taking into consideration for an adequate modelling of the FT-2 experiments.

To take into account the evolution of the EDF, plasma parameters and electric field radial profile we modified the FRTC code to include a relatively weak inductive electric field using a time-dependent solution of the Fokker-Planch equation (1) for the EDF. This version of the FRTC was coupled with the ASTRA. The whole scheme works as follows. At a given ASTRA time step the FRTC reads equilibrium, plasma parameters and electric field radial profile from ASTRA, calculates electron distribution function of fast electrons at this time step, finds the corresponding LH driven current and power absorption density profiles and returns these profiles into ASTRA as external current and heat source, which are then used by ASTRA for equilibrium and plasma parameters recalculation. So, the whole scheme provides self-consistent simulations of plasma shots, which can be compared with experimental results. The very similar scheme was used in the paper [11] where TRANSP and CQL3D were utilized instead of ASTRA and FRTC.

In Fig. 8 you can see the first modelling result for the FT-2 shot \#041514_35 obtained by using the above scheme. It is seen from the Fig. 8 that the nonstationary approach gives a rather good agreement between experimental and modelling results. Calculated loop voltage generally follows the experimental time trace. Agreement between modelling and experiment can be improved, for instance, by introducing a small change to the power sharing in the initial $N_{\|}$spectrum lobes or a small variation to the spectrum form, because we actually know this spectrum only approximately.

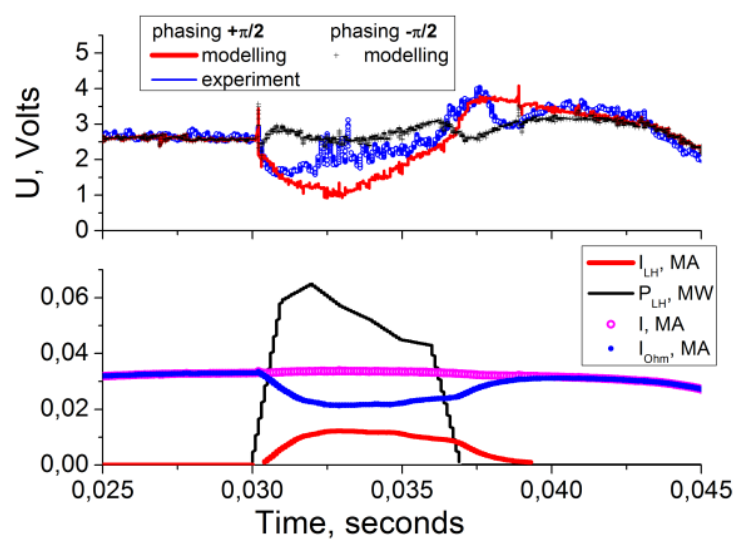

Fig. 8. Comparison of experimental and modelled loop voltage for FT-2 tokomak LHCD experiment, shot \#041514_35. 
The last figure Fig. 9 of this paper shows some radial profiles of the FT-2 shot\#041514_35 at time slice $\mathrm{t}=0.032$ seconds plus the $\mathrm{LH}$ driven current profile given in Fig. 7a. Comparing this radial profile (solid curve, $\mathrm{I}_{\mathrm{LH}}=27.3 \mathrm{kA}$ ) with the corresponding self-consistently modelled LH current profile (circles, $\mathrm{I}_{\mathrm{LH}}=11 \mathrm{kA}$ ), one can estimate the difference between the time-dependent approach and the steady-state EDF application for the LHCD simulations in the case when $t_{\text {asymp }}$ is comparable with the LH power impulse duration.
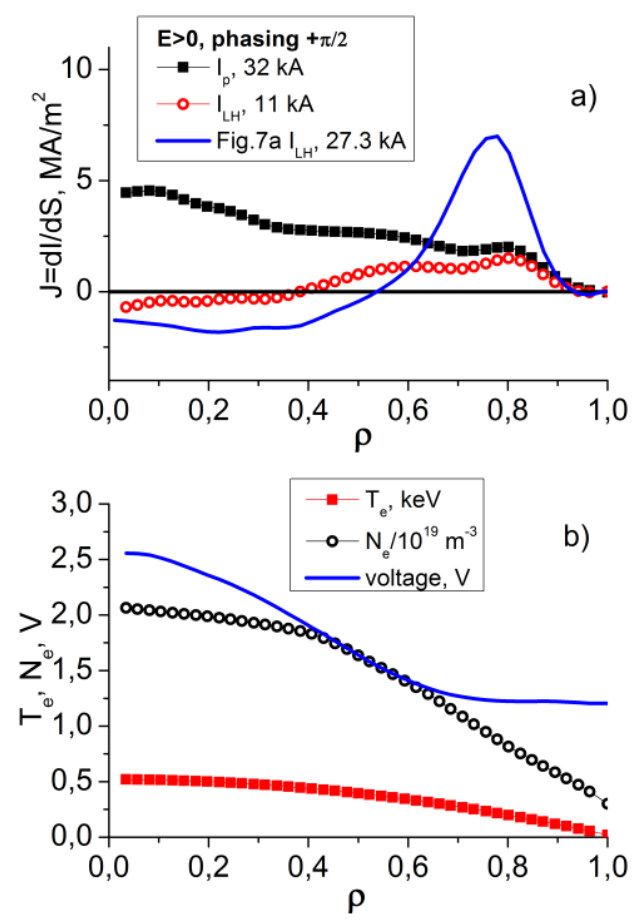

Fig. 9. Radial profiles for the shot \#041514_35, LHCD experiment in the FT-2 tokomak.

a) Solid squares - plasma current, cycles - driven current, solid curve - driven current calculated by FRTC using the steadystate formulae (2-5) for the EDF.

b) Solid squares - plasma temperature, cycles - plasma density, solid curve - voltage radial profile.

\section{Summary}

Formulae (2-4) and the conclusion that nonstationary approach should be used in many cases in the LHCD modelling for adequate explanations of experimental results are the main results of this paper. Also a new scheme for the LHCD modelling is developed. A good applicability of the scheme is demonstrated using an example of an FT-2 shot.

The financial support of the RSF grant 16-12-10043 and the Ioffe Institute is acknowledged.

\section{References}

1. G.V. Pereverzev and P.N. Yushmanov, ASTRA Automated System for TRansport Analysis IPPReport IPP 5/98, (2002)
2. S. I. Lashkul et al. Plasma Physics Reports, 36(9), 751 (2010)

3. C.F.F. Karney and N.J. Fisch, Phys.Fluids, 22(9), 1817 (1979)

4. N.J. Fisch, Rev. Mod. Phys. 59(1), 175 (1987)

5. A.A. Vedenov, in Reviews of Plasma Physics, edited by M.A. Leontovich (Consultants Bureau, New York, 1967), 3, 229

6. E.M. Lifshitz, L.P. Pitaevskii, Physical Kinetics, 10 (Pergamon Press., 1st ed., 1981)

7. A.V. Gurevich, Soviet Physics JETP, 12(8), 904 (1960)

8. Esterkin A R and Piliya A D, Nucl. Fusion, 38, 1501 (1996)

9. Piliya A D and Saveliev A N, Preprint JET-R, 01 (1998)

10. M. A. Irzak and O. N. Shcherbinin, Nucl. Fusion 35, 1341-56 (1995)

11. F. Poli et al, Plasma Physics and Controlled Fusion 58, 095001 (2016) 